\title{
Constraints on the Formation Regions of Comets from their D:H Ratios
}

\author{
JONATHAN HORNER \\ Physikalisches Institut, University of Bern, Sidlerstrasse 5, CH-3012, Bern, Switzerland \\ (E-mail: jonathan.horner@phim.unibe.ch) \\ OLIVIER MOUSIS \\ Observatoire de Besançon, CNRS-UMR 6091, BP 1615, 25010, Besançon Cedex, France \\ FRANCK HERSANT \\ LESIA, Observatoire de Meudon, CNRS-UMR 8109, 5 Place Jules Janssen, 92195, Meudon \\ Cedex, France
}

(Received 28 December 2005; Accepted 7 June 2006)

\begin{abstract}
Studies of the D:H ratio in $\mathrm{H}_{2} \mathrm{O}$ within the Solar nebula provide a relationship between the degree of enrichment of deuterium and the distance from the young Sun. In the context of cometary formation, such models suggest that comets which formed in different regions of the Solar nebula should have measurably different $\mathrm{D}: \mathrm{H}$ ratios. We aim to illustrate how the observed comets can give information about the formation regions of the reservoirs in which they originated. After a discussion of the current understanding of the regions in which comets formed, simple models of plausible formation regions for two different cometary reservoirs (the Edgeworth-Kuiper belt and the Oort Cloud) are convolved with a deuterium-enrichment profile for the pre-solar nebula. This allows us to illustrate how different formation regions for these objects can lead to great variations in the deuterium enrichment distributions that we would observe in comets today. We also provide an illustrative example of how variations in the population within a source region can modify the resulting observational profile. The convolution of a deuterium-enrichment profile with examples of proto-cometary populations gives a feel for how observations could be used to draw conclusions on the formation region of comets which are currently fed into the inner Solar system from at least two reservoirs. Such observations have, to date, been carried out on only three comets, but future work with instruments such as ALMA and Herschel should vastly improve the dataset, leading to a clearer consensus on the formation of the Oort cloud and Edgeworth-Kuiper belt.
\end{abstract}

Keywords: Comets: general, Kuiper Belt, Oort Cloud, Solar system: formation

\section{Introduction}

The question of how our Solar system formed is one of great interest. Many models have been proposed over the years (e.g. Lyttleton, 1941; Pendred and Williams, 1968; Oxley and Woolfson, 2004), based on the evidence available at the time. However, the formation mechanism is debated as strongly now as at any point in history. The discovery of the first extra-solar planets has 
thrown open the field of planetary formation, with many unexpected results being obtained (Mayor and Queloz, 1995; Perryman, 2000). At the same time, the discovery of the Edgeworth-Kuiper belt and Scattered disk (Jewitt and Luu, 1993) beyond Neptune, together with the detailed study of their dynamics (e.g. Malhotra et al., 2000; Levison and Morbidelli, 2003; Emel'yaneko et al. 2004), has placed some new constraints on the migration of the outer planets.

One piece of evidence which may allow us to differentiate between different models of planet formation relates to comets. These icy bodies are likely to retain the isotopic composition they acquired when they formed (Drouart et al. 1999). As such, it may be possible to determine, from their observed properties, their formation region. Within our Solar system, there are two main reservoirs for these cometary bodies - the Edgeworth-Kuiper belt and Scattered disk, just beyond Neptune, and the Oort cloud, which reaches out to approximately half-way to the nearest star (Oort, 1950; Dones et al. 2004). It is quite possible that the comets which make up these reservoirs formed in different regions within the planetary nebula, and therefore comets from these different regions may have different isotopic compositions. To date, the isotopic composition of water has only been measured in three comets - 1P/Halley, 1996 B2 (Hyakutake) and 1995 O1 (Hale-Bopp). In the context of the currently accepted formation scenarios for comets, the similarity of measurements of $\mathrm{D}: \mathrm{H}$ ratios in $\mathrm{H}_{2} \mathrm{O}$ in these three objects (see Table I) should be considered as fortuitous and can be taken as reflecting formation in a similar part of the Solar nebula.

Using a deuterium-enrichment profile, which offers a relationship between the deuterium to hydrogen (henceforth $\mathrm{D}: \mathrm{H}$ ) ratio incorporated in the water within planetesimals and their formation location in the Solar nebula (Drouart et al. 1999; Mousis et al. 2000; Hersant et al., 2001), we examine the possible effect that formation in different regions could have on the values of this ratio observed in comets today. While there are other compounds which contain deuterium, water is the ideal choice for such a study. It represents the main deuterated reservoir remaining in the Solar system, and is also the main volatile component in cometary bodies. We illustrate how such

TABLE I

Deuterium measurements in $\mathrm{H}_{2} \mathrm{O}$ in different comets

\begin{tabular}{lll}
\hline Comet & D:H & Reference \\
\hline 1P/Halley & $\left(3.08^{+0.38}{ }_{-0.53}\right) \times 10^{-4}$ & Balsiger et al. (1995) \\
1P/Halley & $(3.06 \pm 0.34) \times 10^{-4}$ & Eberhardt et al. (1995) \\
C/1996 B2 (Hyakutake) & $(2.9 \pm 1.0) \times 10^{-4}$ & Bockelée-Morvan et al. (1998) \\
C/1995 O1 (Hale-Bopp) & $(3.3 \pm 0.8) \times 10^{-4}$ & Meier et al. (1998) \\
\hline
\end{tabular}


a model, when combined with observations, can lead to conclusions on the regions in which different cometary populations were born.

In Section 2, a brief description of the model used to obtain the behaviour of the $\mathrm{D}: \mathrm{H}$ ratio in $\mathrm{H}_{2} \mathrm{O}$ in the Solar nebula is presented, along with a discussion of the influence of turbulent diffusion on the calculated enrichment profile. In Section 3, the leading models describing the formation of comets are discussed, and in Section 4, simple examples are used to illustrate how variations in the formation regions of the comets could be reflected in the measured D:H observed in comets today. In Section 5, we conclude with a discussion of the implications of such work in light of future measurements.

\section{The Evolution of the D:H Ratio in $\mathrm{H}_{2} \mathrm{O}$ in the Solar Nebula}

\subsection{Isotopic Fractionation of Deuterium}

The main reservoir of deuterium in the Solar nebula was molecular hydrogen (HD vs. $\mathrm{H}_{2}$ ). Ion-molecule reactions in the interstellar medium (see e.g. Brown and Millar, 1989) result in the fractionation of deuterium between deuterated species. Consequently, in the pre-solar cloud, such fractionation was present, resulting in heavier molecules being enriched. Water was the second most abundant hydrogen bearer in the solar nebula, as it is in our current Solar system, and therefore became the second largest deuterium reservoir.

In the Solar nebula the isotopic fractionation of deuterium between water and hydrogen followed the reversible reaction (Geiss and Reeves, 1981)

$$
\mathrm{H}_{2} \mathrm{O}+\mathrm{HD} \rightleftharpoons \mathrm{HDO}+\mathrm{H}_{2}
$$

At low temperatures, this reaction favours the concentration of deuterium in HDO, but the reaction kinetics at such temperatures tend to inhibit such enrichment of deuterium in water. The enrichment factor, $f$, which results from the exchange between HD and HDO is defined as the ratio of $\mathrm{D}: \mathrm{H}$ in the considered deuterated species to that in molecular hydrogen (the protosolar value). As a result, for water we have:

$$
f=\frac{1 / 2}{1 / 2} \frac{\mathrm{HDO} / \mathrm{H}_{2} \mathrm{O}}{\mathrm{HD} / \mathrm{H}_{2}}
$$


We follow the work of Drouart et al. (1999), Mousis et al. (2000) and Hersant et al. (2001) who described the evolution of the deuterium enrichment factor, $f$, in $\mathrm{H}_{2} \mathrm{O}$ within the Solar nebula. These authors physically interpreted the measurements of the D:H ratio in the LL3 meteorites, along with measurements taken of comets 1P/Halley, C/1996 B2 (Hyakutake), and C/1995 O1 (Hale-Bopp) (all three of which share a similar value of D:H in $\mathrm{H}_{2} \mathrm{O}$ ). Time dependent turbulent models of the Solar nebula were then applied which depend on three physical parameters: the initial mass of the nebula $M_{\mathrm{D} 0}$, its initial radius $R_{\mathrm{D} 0}$, and the coefficient of turbulent viscosity $\alpha$ (derived from the prescription of Shakura and Sunyaev (1973)). They calculated $f$ in water with respect to the protosolar value in molecular hydrogen by integrating an equation of diffusion in the Solar nebula, as a function of the heliocentric distance and time. The comparison of the obtained value of $f$ to observations allowed a range of possible values for $M_{\mathrm{D} 0}, R_{\mathrm{D} 0}$, and $\alpha$ to be determined.

This equation of diffusion takes into account the isotopic exchange between $\mathrm{HDO}$ and $\mathrm{H}_{2}$ in the vapour phase, and turbulent diffusion throughout the Solar nebula. The diffusion equation remains valid as long as $\mathrm{H}_{2} \mathrm{O}$ does not condense, which implies that the value of $f$ 'frozen' into microscopic ices is that present at the time and location of condensation. As soon as the grains reach millimetre size, they begin to decouple from the gas as they grow further, leading to the formation of planetesimals. Whatever the subsequent evolution of these bodies, their $\mathrm{D}: \mathrm{H}$ ratio is that of the microscopic grains from which they formed. In this paper, we consider the case where the cometesimals that grew in the Solar nebula were accreted only from icy grains formed locally. This means that the $\mathrm{D}: \mathrm{H}$ ratio in the deuterated ices within comets is that which was present at the time and location at which they condensed.

In these calculations we have only accounted for neutral-neutral reactions. In regions of the nebula where the level of ionisation was high enough, interstellar-like chemistry was certainly an important factor (Aikawa and Herbst, 1999, 2001). However, the formation of the comets occurred in the first few $10^{5}$ years (Mousis et al. 2000), at a time when the nebula was too dense to be completely ionised. Ionisation only occurred at the very surface of the disk and concerned a very small fraction of the nebula (See Fromang et al. 2002 for a discussion about ionisation of disks). Therefore, although ionic chemistry certainly affected the $\mathrm{D}: \mathrm{H}$ fractionation in the Solar system, it was only important in a small fraction of the disk and we neglect this effect in the present work. For more detailed studies, such reactions will need to be taken into account.

Figure 1 shows the variation of $f$ for water trapped within icy grains, as a function of their distance from the Sun, in the case of the maximum-mass Solar nebula (as defined by Mousis et al. 2000; see Table II). Here, as in 


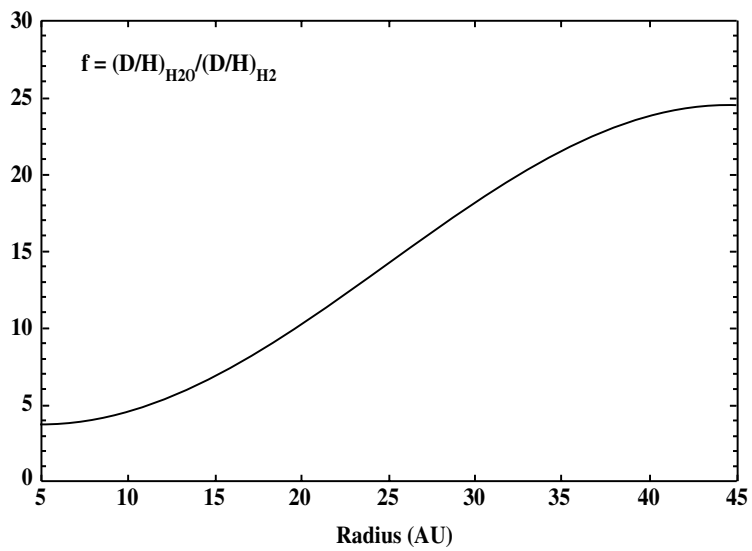

Figure 1. The calculated deuterium enrichment factor, $f$, in $\mathrm{H}_{2} \mathrm{O}$ as a function of heliocentric distance $a$, in the case of the maximum-mass solar nebula model derived by Mousis et al. (2000). The value of $f$ is fixed when the water condenses.

TABLE II

Thermodynamical parameters of the maximum-mass Solar nebula (Mousis et al. 2000)

Parameters

Viscosity parameter $\alpha$

Initial disk's radius $R_{D 0}(\mathrm{AU})$

Initial disk's mass $M_{D 0}\left(M_{\odot}\right)$

0.3

previous work, we assume that $f(R)=31$ at $t=0$ for $\mathrm{D}: \mathrm{H}$ in water. This value corresponds to that measured in the highly enriched component (where $\left.\mathrm{D}: \mathrm{H}=(73 \pm 12) \times 10^{-5}\right)$ found in LL3 meteorites (Deloule et al. 1998) compared to a protosolar value assumed to be $(2.35 \pm 0.3) \times 10^{-5}$ (Mousis et al. 2002). As can be seen, towards the inner and outer edges of the disk, the slope in $f$ is very shallow, while the variation is greatest between 10 and $40 \mathrm{Au}$. Given that this is the most likely formation region for the comets, this diagram illustrates why $f$ may prove a good probe of the mechanisms involved in populating the different cometary reservoirs. This profile is used later, in Section 4.

\subsection{The Role of Turbulent Diffusion}

In this study we neglect the role of diffusion and the transport of icy grains through the nebula resulting from gas turbulence. In particular, our model neglects the possible radial redistribution of solid material due to gas drag, an effect that could have been substantial in the early Solar system (e.g. Cuzzi 
and Zahnle 2004). Turbulent diffusion mixes grains together, thereby producing a flatter profile for the enrichment vs. distance relation. When grains condense from the nebula, they have a $\mathrm{D}: \mathrm{H}$ ratio which is given by the D-enrichment factor (see Figure 1). Once condensed, they initially remain coupled to the gas dynamics. Consequently they diffuse under the action of turbulent mixing. As these icy grains agglomerate to form larger objects, the net effect of the motion of the gas is reduced, and eventually these objects become decoupled from the gas. The distribution of $\mathrm{D}: \mathrm{H}$ in Solar system objects is therefore the result of the full dynamics and growth of grains in the nebula. The computation of these combined effects remains an arduous task and is beyond the scope of this paper.

However, instead of attempting to compute the exact distribution of D:H in objects, we can use observed distributions to estimate the effect of turbulent diffusion. Measured D:H distributions are, for the moment, only available for meteorites. An example of observed distributions is given in Figure 2, adapted from the compilation of Robert et al. (2000).

While the observed distribution gives a nice illustration of how complex the transport of grains within the disk can be, such a distribution can also be used to determine the properties of average transport in the nebula. The dispersion of the $\mathrm{D}: \mathrm{H}$ distribution in chondrules around the mean chondritic value appears to be \pm 2 around the mean chondritic value (in $f$ ). The carbonaceous meteorites show a similar behaviour. However the very flat distribution seen for the clays in the LL3 chondrites reflects their different origin (since clays require liquid water in order to form). The asymmetry of the distribution towards larger $\mathrm{D}: \mathrm{H}$ is a consequence of the mass distribution in the nebula, with increasing mass being present in annuli at larger heliocentric distances. Consequently, using Figure 1, the dispersion of \pm 2 around the mean chondritic value of $\sim 6$ in enrichment translates to a "range" of turbulent mixing of about \pm 5 AU around the mean condensation distance of the grains.

Without being precise, this illustrates that icy objects that formed at different distances should have measurably different $\mathrm{D}: \mathrm{H}$ ratios. This implies that, even though the effect of turbulent diffusion is not considered in Figure 1 , this D-enrichment profile meets all our requirements to discuss the plausible variation of $\mathrm{D}: \mathrm{H}$ in comets as a function of their formation regions in the Solar nebula.

\section{The Formation of the Oort Cloud, Edgeworth-Kuiper Belt and the Scattered Disk}

Given a relationship between the D-enrichment $(f)$ with changing location in the Solar nebula, it is clear that measurement of this ratio within the comae 


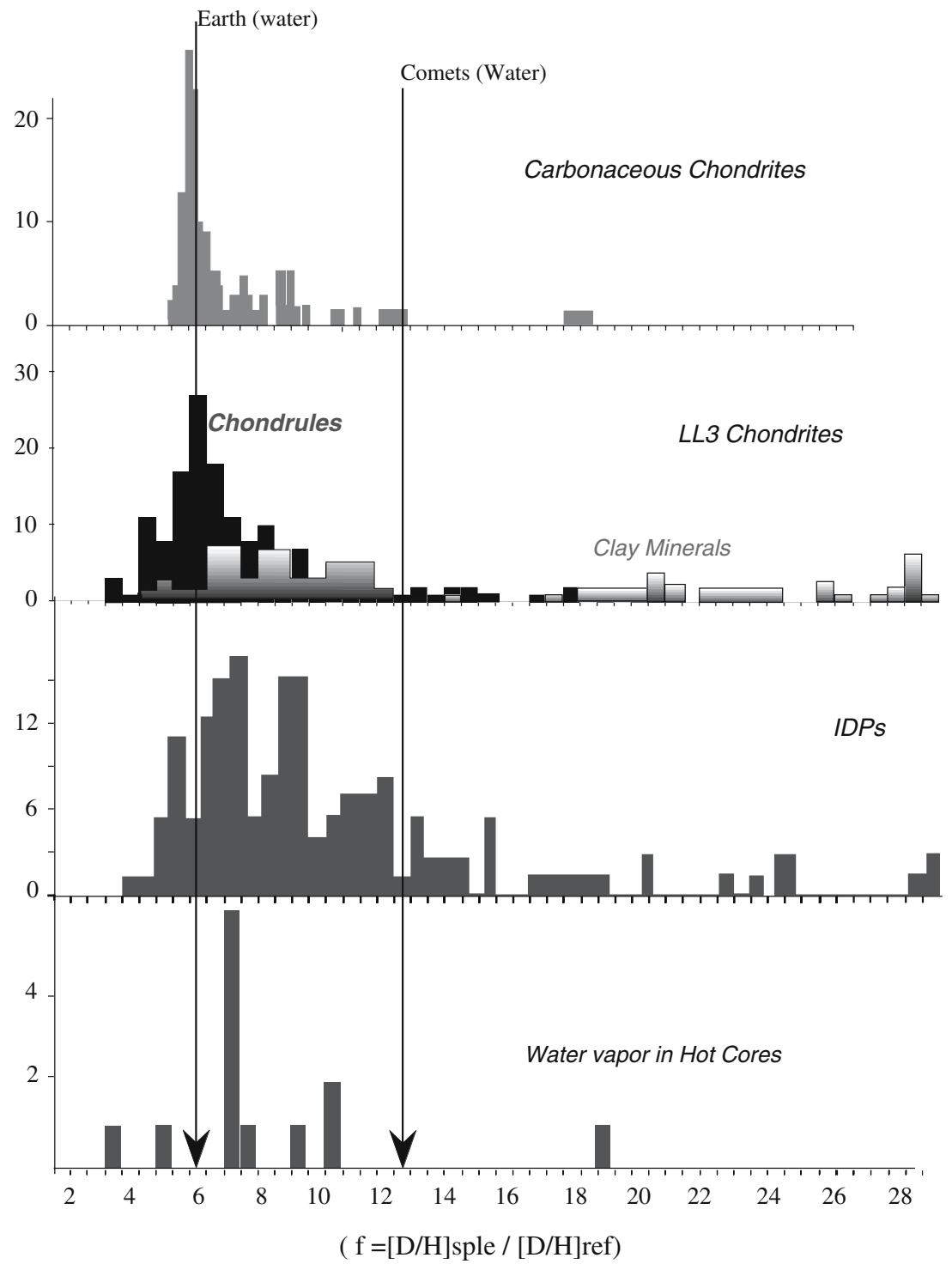

Figure 2. D:H enrichment histogram showing the enhancement over the Solar value of the $\mathrm{D}: \mathrm{H}$ ratio in Carbonaceous chondrites, LL3 chondrites and interplanetary dust particles. For comparison, the observed values from interstellar molecular clouds are show in the lowest panel (private communication: updated from Robert et al. 2000).

and tails of comets could be used to infer the location within the nebula at which the comet formed. What, then, can we infer from such measurements about the formation of the Oort cloud and the Edgeworth-Kuiper belt? 


\subsection{The Edgeworth-Kuiper Belt}

It is almost universally agreed that the majority of the bodies within the Edgeworth-Kuiper belt formed at a distance further from the Sun than that of Neptune (which, prior to migration, could have been located at a semi-major axis of between 15 and $20 \mathrm{Au}$ ) (Gladman, 2005). As Neptune migrated outwards, it swept these bodies along with it, in a manner similar to the sweeping of dust before a broom. From the relationship presented above, it is clear that such bodies would be expected to have relatively high $f$, in the main, reflecting their formation at distances of 15-30 Au or more.

Objects from the Edgeworth-Kuiper belt are one possible source for the Jupiter Family comets (JFCs) (Horner et al. 2004a, b). Objects ejected from this region by dynamical or collisional processes can work their way inwards through the Centaur region, arriving in the inner Solar system whilst under Jupiter's control. Therefore, if the Edgeworth-Kuiper belt is the principle source of the JFCs, we would expect them to be dominated by objects with equally high $f$, reflecting their formation in the outer Solar system.

\subsection{The Scattered Disk}

The Scattered Disk contains a population of objects which lie beyond Neptune, but on significantly more eccentric orbits than the Edgeworth-Kuiper belt objects. The division of objects between the Edgeworth-Kuiper belt and the Scattered Disk is not yet a well defined process - the exact definition of the two regions is still open to some debate. Here, we follow Morbidelli and Levison (2004), who define the Scattered Disk as 'the region of the orbital space that can be visited by bodies that have encountered Neptune within a Hill's radius at least once during the age of the Solar system, assuming no substantial migration of the planetary orbits'. Under this definition, it is clear that the Scattered Disk, rather than the Edgeworth-Kuiper belt (which is effectively the stable counterpart to this Disk) would be the primary source of the Centaurs and thus the JFCs (Emel'yaneko et al. 2005). The Scattered Disk is expected to contain more cometary bodies which formed within the orbit of Neptune than the Edgeworth-Kuiper belt. However, it is still likely that the majority of objects within the Disk formed at, or beyond, Uranus and Neptune. Therefore, should the JFC population come primarily from this region, we would expect that the majority of the objects there would still have large $f$. 


\subsection{The Oort Cloud}

The formation of the Oort cloud is, when viewed in the light of the model described above, a region of particular interest. Theories of the main source region for the Oort cloud vary, from the suggestion that the bulk of objects therein formed near Jupiter and Saturn (Whipple, 2000; Goldreich et al. 2004), and were ejected by these massive planets, to others where the vast majority of objects in the Oort cloud formed further out, and were ejected primarily by Uranus and Neptune (Jakubík and Neslušan, 2002). The work of Fernández et al. (2004) suggests that the Oort cloud is being replenished by objects from the Scattered Disk. This means that the objects which make up the Oort cloud would be expected to have a diverse range of $f$. However, the study of the variation of $f$ in new comets could help to answer the main formation region of the Oort cloud - if the bulk are found to have low $f$ (such as was suggested for C/ 1999 S4 Linear by Mumma et al. (2001)) then it could be inferred that they formed in the inner parts of the outer Solar system, while if the bulk are found to possess high $f$ (such as C/ $1995 \mathrm{O} 1$ Hale-Bopp) this would argue for a formation region further out (Mousis et al. 2000). It is quite likely that there will be a larger range of $f$ in the Oort cloud comet flux than in the JFC population, but information on the primary formation region would prove useful in constraining things such as the environment in which the Solar system formed - whether it was in a dense stellar cluster, or a much sparser region (Fernández, 1997).

\subsection{The Jovian Trojans}

It is suggested (e.g. Marzari et al. 1995) that as many as $10 \%$ of the objects within the JFC population may actually originate from the Trojan asteroid swarms, which lie within the 1:1 mean-motion resonance of Jupiter. The Trojans, in the main, are thought to represent a reservoir of objects which have resided in this region since the formation of the Solar system (Shoemaker et al. 1989; Morbidelli et al., 2004), rather than being objects captured temporarily from the Asteroid belt or Jupiter Family. Temporary capture of cometary bodies into the Trojan region can occur (Horner and Evans, 2006), but such interlopers are likely to only make up a small fraction of the total Trojan population. It is possible, however, that the bulk of the Jovian Trojans currently observed may have originated much further from the Sun than their present location, and have been captured as a by-product of the later stages of planetary migration (Morbidelli et al., 2005).

Whichever mechanism created the clouds we observe today, objects in the Trojan clouds can be ejected by both dynamical and physical processes, and a 
common destination for such ejected particles would be the JFC population (Karlsson, 2004).

JFC comets originating in the Trojan region could, in theory, display $f$ values much lower than those for bodies which originated in the Edgeworth-Kuiper belt or the Scattered disk, if they did indeed form in the vicinity of Jupiter. This, in turn, would suggest that the JFCs may even display a bimodal $f$ distribution, with the heights of the two peaks determined by the fraction of JFCs which originated in the Trojan region, and the widths of the peaks dependent on the amount of mixing of cometary bodies in the Solar nebula. Should the second hypothesis prove correct (the capture of the Trojans from a more distant source population), then this would obviously not be the case.

\section{Illustrating the Effect of Changing Source Populations}

In order to illustrate the changes that the differing views of comet formation will wreak on the observed distribution of $f$ values in comets, and hence to show how observations of these values can aid us in the determination of these formation locations, we present four simple figures (Figure 3). In these diagrams, the $f$ vs. $a$ relationship, shown in Figure 1, is combined with particularly simple assumed initial cometary populations, allowing the calculation of the resulting distribution of $f$ values which would be observed. We illustrate the concept by showing two populations. One, which can be considered to be analogous to the objects which formed the Edgeworth-Kuiper belt, is always assumed to have formed between 20.1 and $35.1 \mathrm{Au}$ (for clarity, this is shifted slightly from 20 to 35 to prevent overlap with the other population), whilst the other, which may be taken to represent the objects which now make up the Oort cloud, is shown forming in different regions. In the first three cases, the assumed 'Edgeworth-Kuiper' population was also taken to be $10 \%$ lower than that for the 'Oort cloud'. This, again, was merely done as an aid to visual clarity.

For each of the four diagrams, the upper panel shows the two initial populations used, while the lower shows the resulting $f$ distribution (normalised so that the sum over all $f$-values equals one for each of the two populations shown).

In the first diagram (plots a), the 'Oort cloud' population is taken as forming solely between 5 and $20 \mathrm{Au}$, and hence has no overlap with the region of the Edgeworth-Kuiper belt's formation. In this plot (together with the next two) the most simple possible particle distribution is assumed - a constant number of particles per unit $a$ in the region of formation, and zero particles elsewhere. In the resulting $f$ distribution plot, two features are clearly seen. Firstly, there is a sharp spike in the 'Oort-cloud' section of the distribution at around $f=5$. This is due to the plateau in the $f$-values in the inner 

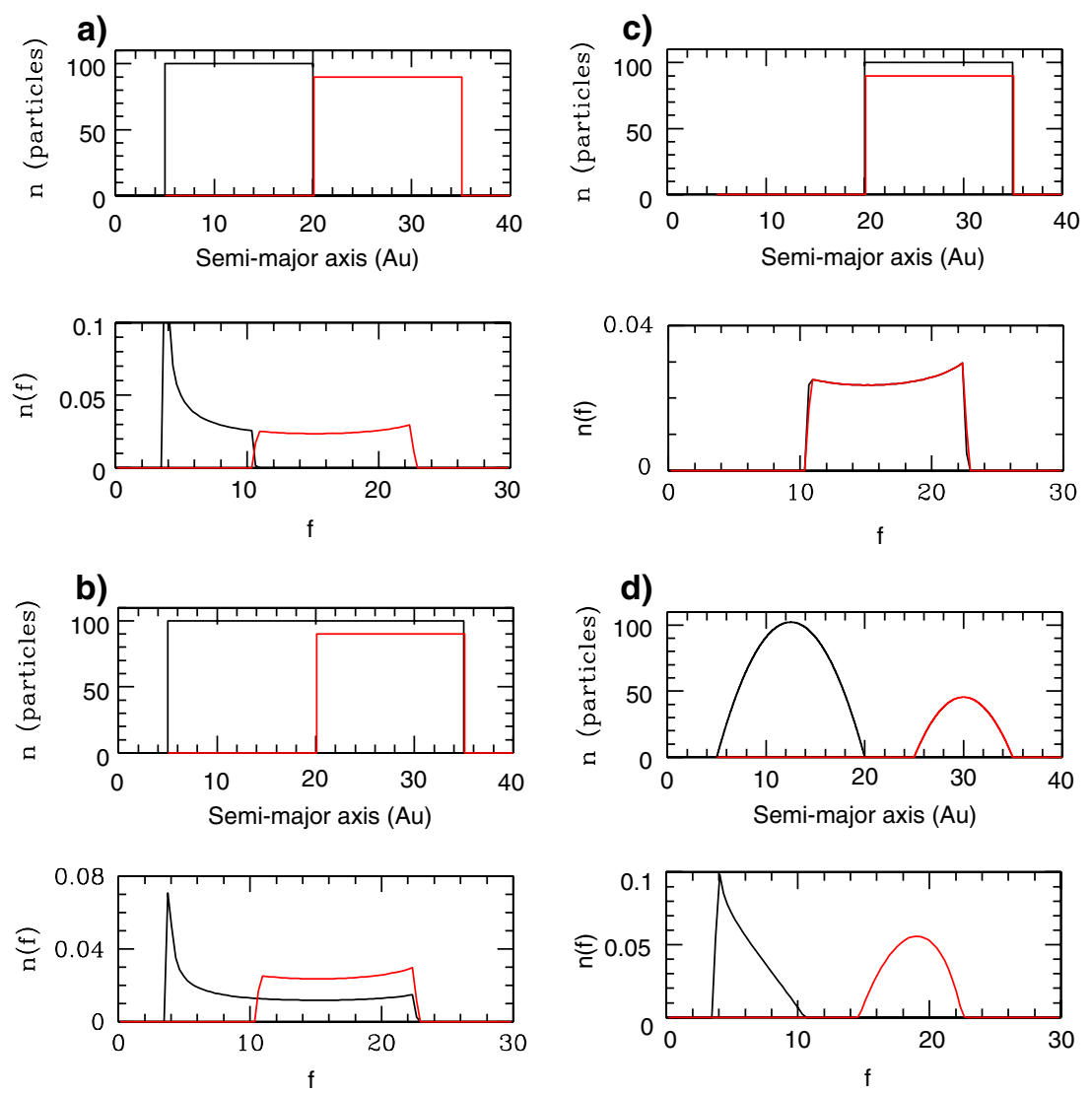

Figure 3. The panels show different examples of how the formation regions of comets affect their observed $f$ distributions. In each of the four diagrams, the upper shows the used population distributions, and the lower the resulting $f$ distribution that would be observed. The red line shows our example 'Edgeworth-Kuiper' objects, and the black the 'Oort cloud' bodies.

region of the outer Solar system. The second feature is that the objects in the 'Edgeworth-Kuiper belt' region have a distinctly different $f$ profile to those which went to form our 'Oort cloud'. It is clear from this that, if the Oort cloud and Edgeworth-Kuiper belt objects had formed in different locations, their observed $f$ distributions would be measurably different.

In the second diagram (b), the 'Oort cloud' population (now taken as forming between 5 and $35 \mathrm{u}$ ) has a large amount of overlap with those particles which make up our 'Edgeworth-Kuiper belt'. However, despite this overlap, the two resulting $f$ distributions are again noticeably different. Therefore, even if there was some degree of mixing between the two populations, differences would still be observed between them if they also encompassed different formation regions. 
The third diagram (c) shows the situation when the 'Oort cloud' and 'Edgeworth-Kuiper belt' populations formed in exactly the same region, with the same distribution. In this case, the resulting $f$ distributions are identical, and the populations look the same in $f$ space.

The fourth, and final diagram (d) shows how studying the $f$ distribution may also inform us about the initial distribution of objects within their formation region. In this diagram, rather than taking the uniform distribution used in the first three, a slightly more complicated distribution was used. In both cases (the 'Oort cloud' and 'Edgeworth-Kuiper belt' objects), the formula used to determine the initial population as a function of $a$ was as follows:

$$
n=200 \times\left(\left(a-a_{\text {in }}\right) *\left(a_{\text {out }}-a\right)\right)
$$

Here, $n$ is the number of objects located within the semi-major axis band centred on $a$, and $a_{\text {in }}$ and $a_{\text {out }}$ give the locations of the inner and outer edges of the disk of comets, respectively. In this plot, it can be seen that the shape of the initial distribution of comets has a radical effect on the shape of the resulting $f$ distribution, and hence, that knowledge of the $f$ distribution may be used to draw inferences on the initial population distributions within the regions of comet formation, beyond the simple location of those regions. It should be noted that, in this final plot, our Edgeworth-Kuiper belt objects were taken as forming between 25 and $35 \mathrm{Au}$. This was purely to remove any overlap between the two populations, so that the effect of their shape on the $f$ distribution could be more clearly seen.

It is therefore clear that, given initial distributions for the comets which make up the different reservoirs within our Solar system, it is possible to calculate a theoretical $f$ distribution for those populations. It is also clearly feasible to work the other way. Given enough observed $f$ values for comets of different classes, one could work backwards to calculate where those classes were predominantly formed. Whilst the real initial distributions for the objects now in the Edgeworth-Kuiper belt and the Oort cloud are no doubt significantly more complicated than those used in illustrating the principle, it seems that future measurements of $f$ values in a variety of comets could lead to a much greater understanding of the regions in which they formed, and therefore of the environment in, and around, our Solar system at the time of its birth.

\section{Conclusions}

The variation of the deuterium-enrichment profile in $\mathrm{H}_{2} \mathrm{O}$ as a function of position in the Solar nebula has been widely studied (Drouart et al. 1999; 
Mousis et al. 2000; Hersant et al. 2001). This leads to the conclusion that the further one travels from the Sun in this nebula, the greater the enhancement in the D:H ratio. This feature is particularly strong between 10 and $40 \mathrm{Au}$, encompassing neatly the region in which most cometary bodies are believed to have formed. In this paper, we suggest that the measurement of this $\mathrm{D}: \mathrm{H}$ ratio in comets of different families could help answer the questions which still remain about their formation regions, together with the population distributions therein. Illustrative examples are presented, highlighting how different source regions, together with differing initial distributions within these regions, would affect the shape of the distribution of $\mathrm{D}: \mathrm{H}$ ratios that would be observed in comets today. Importantly, the conclusion that measurements of $\mathrm{D}: \mathrm{H}$ in multiple comets would help refine the theories on their formation is independent of the values resulting from the initial model. Regardless of the initial model of the thermodynamical evolution of the Solar nebula that is used, the general behaviour would be the same, with merely the D:H values measured being raised or lowered accordingly.

Until recently, radio observations of the $\mathrm{D}: \mathrm{H}$ ratio in comets have been particularly difficult to carry out, and accurate values have only been determined for 3 comets (1P/Halley, 1995 O1 Hale-Bopp and 1996 B2 Hyakutake (Altwegg and Bockelée-Morvan, 2003)). The values obtained for these three objects are very similar. This may infer that they formed in similar regions within the Solar nebula, but more measurements are needed to provide any kind of reasonable sample of the cometary population. In the near future, two new projects will come on-line (ALMA and Herschel), making such measurements easier, and hence greatly enlarging the known dataset. In fact, such measurements of Edgeworth-Kuiper belt objects and Jupiter family comets are one of the main objectives of the Herschel mission, and the mission will provide guaranteed time for such measurements. We expect that these observations could prove of vital importance in finally settling such questions as that of the main initial source region of the Oort cloud, and in turn will help to understand the environment in which the Solar system was created.

\section{Acknowledgements}

We thank F. Robert for providing Figure 2, and JH acknowledges the support of the Swiss National Science Foundation. 


\section{References}

Aikawa, Y. and Herbst, E.: 1999, ApJ 526, 314.

Aikawa, Y. and Herbst, E.: 2001, $A \& A$ 371, 1107.

Altwegg, K. and Bockelée-Morvan, D.: 2003, Space Sci. Rev. 106, 139.

Balsiger, H., Altwegg, K., and Geiss, J.: 1995, JGR 100, 5827.

Bockelée-Morvan, D., Gautier, D., and Lis, D. C. et al.: 1998, Icarus 133, 147.

Brown, P. D. and Millar, T. J.: 1989, MNRAS 237, 661.

Cuzzi, J. N. and Zahnle, K. J.: 2004, ApJ 614, 490.

Deloule, E., Doukhan, J.-C., and Robert, F.: 1998, Geochim. Cosmochim. Acta 62, 3367.

Dones, L., Weissman, P. R., Levison, H. F. et al.: 2004, ASP Conf. Ser. 323: Star Formation in the Interstellar Medium: In Honor of David Hollenbach, 323, 371

Drouart, A., Dubrulle, B., and Gautier, D. et al.: 1999, Icarus 140, 129.

Eberhardt, P., Meier, R., and Krankowsky, D. et al.: 1995, A\&A 302, 301.

Emel'yanenko, V.V., Asher, D.J., and Bailey, M.E.: 2004, MNRAS 350, 161.

Emel'yanenko, V.V, Asher, D.J., and Bailey, M.E.: 2005, MNRAS 361, 1345.

Fernández, J.A.: 1997, Icarus 129, 106.

Fernández, J.A., Gallardo, T., and Brunini, A.: 2004, Icarus 172, 372.

Fromang, S., Terquem, C., and Balbus, S.A.: 2002, MNRAS 329, 18.

Geiss, J. and Reeves, H.: 1981, $A \& A$ 93, 189.

Gladman, B.: 2005, Science 307, 71.

Goldreich, P., Lithwick, Y., and Sari, R.: 2004, ApJ 614, 497.

Hersant, F., Gautier, J.-M., and Huré, D: 2001, ApJ 554, 391.

Horner, J., Evans, N. W., and Bailey, M. E.: 2004a, MNRAS 354, 798.

Horner, J., Evans, N. W., and Bailey, M. E.: 2004b, MNRAS 355, 321.

Horner, J. and Evans, N. W.: 2006, MNRAS 367, L20.

Jakubík, M., \& Neslušan, L. 2002, ESA SP-500: Asteroids, Comets, and Meteors: ACM 2002, 441

Jewitt, D. and Luu, J.: 1993, Nature 362, 730.

Karlsson, O.: 2004, $A \& A$ 413, 1153.

Levison, H. F. and Morbidelli, A.: 2003, Nature 426, 419.

Lyttleton, R.A.: 1941, ApJ 93, 267.

Malhotra, R., Duncan, M. J., and Levison, H. F. 2000, Protostars and Planets IV, 1231

Mayor, M. and Queloz, D.: 1995, Nature 378, 355.

Marzari, F., Farinella, P., and Vanzani, V.: 1995, A\&A 299, 267.

Meier, R., Owen, T. C., and Matthews, H. E. et al.: 1998, Science 279, 842.

Morbidelli, A. and Levison, H.F.: 2004, AJ 128, 2564.

Morbidelli, A., Levison, H.F., and Tsiganis, K. et al.: 2004, AAS/DPS Meeting 36, 40.03.

Morbidelli, A., Levison, H. F., Tsiganis, K., and Gomes, R.: 2005, Nature 435, 462.

Mousis, O., Gautier, D., and Bockelée-Morvan, D. et al.: 2000, Icarus 148, 513.

Mousis, O., Gautier, D., and Coustenis, A.: 2002, Icarus 159, 156.

Mumma, M. J., Russo, N. D., and DiSanti, M. A. et al.: 2001, Science 292, 1334.

Oort, J.H.: 1950, Bull. Astron. Inst. Neth. 11, 91.

Oxley, S. and Woolfson, M.M.: 2004, MNRAS 348, 1135.

Pendred, B. W. and Williams, I. P.: 1968, Icarus 8, 129.

Perryman, M. A. C.: 2000, Rep. Prog. Phys. 63, 1209.

Robert, F., Gautier, D., and Dubrulle, B.: 2000, Space Sci. Rev. 92, 201.

Shakura, N. I. and Sunyaev, R. A.: 1973, $A \& A$ 24, 337.

Shoemaker, E. M., Shoemaker, C. S., and Wolfe, R. F.: 1989, Asteroids II, 487

Whipple, F. L.: 2000, Planet. Space Sci. 48, 1011. 